\title{
Interconnection Contracts between Service and Content Provider with Partial Cloud Migration
}

\author{
Branka D. Mikavica ${ }^{1}$, Aleksandra M. Kostic-Ljubisavljevic ${ }^{1}$ \\ ${ }^{I}$ Faculty of Transport and Traffic Engineering, University of Belgrade, \\ Vojvode Stepe 305, 11000 Belgrade, Serbia \\ b.mikavica@sf.bg.ac.rs
}

\begin{abstract}
This paper addresses interconnection contracts in content provisioning scheme with partial cloud migration. Model for traffic workload of such scheme based on content popularity factor is introduced. Monte Carlo simulation for bandwidth demand estimation is used. Cost analysis of content provider's resources utilization regarding average requests' rejection rate is observed. The performance analysis of content provider's incentive for partial cloud migration is applied. Three interconnection contracts are analysed: Revenue Sharing, Cost Sharing and Wholesale Price contract. Obtained results show that partial cloud migration can reduce both content provider's costs and average requests' rejection rate. Under observed constellation, Revenue Sharing contract may represent satisfying solution for both providers.
\end{abstract}

Index Terms-Clouds; content distribution networks; contracts; interconnection.

\section{INTRODUCTION}

The permanent growth of Internet traffic, caused by emerging high bandwidth demanding contents such as video on demand, High Definition Television (HDTV), real time video, online gaming, file sharing and cloud computing, is supported by bandwidth improvement of all participants in content provisioning process. It includes content providers, service providers, Content Delivery Network (CDN) and customers [1]. Participating with $80 \%-90 \%$ of overall global Internet traffic, video content distribution is the leading issue of bandwidth requirements [2], [3]. In bandwidth demand estimation video content popularity factor is an important parameter and has to be included in network dimensioning process. Video content popularity factor can be described as frequency of access to certain video content [4]. Several observations on the suitable video content popularity distribution can be found in the literature: Zipf [5], [2], Zipf-Mandelbrot [6], stretched exponential [7], Zipf with exponential cut-off tail [8], etc.

Appropriate bandwidth demand estimation is of great importance for addressing resource investment. Once bandwidth demand is estimated, network resources can be allocated in a way that costs can be minimized. Since peak bandwidth demand changes during the day, provisioning of self-owned resources that satisfy peak bandwidth demand is

Manuscript received 5 April, 2016; accepted 25 October, 2016.

This research was partially funded by a grant (No. TR 32025) from the Ministry of Education, Science and Technological Development of Serbia. cost ineffective. These resources would be underutilized in the periods of low or normal traffic load. On the other hand, under-provisioning leads to rejection of requests. In order to minimize costs and improve Quality of Service (QoS) and hence Quality of Experience (QoE) of their customers, content providers need to consider using resources of cloud providers. Cloud providers maintain large-scale data centres to offer storage and computational resources at a relatively low cost [9]-[11]. These providers enable different pricing plans, such as reservation, on-demand and spot market [12]. On-demand pricing plan enables cloud providers' customers to pay only for utilized resources on the hourly basis. In the reservation plan, cloud providers' customers pay an upfront reservation fee in order to reserve cloud resources for a specific period of time. In exchange, they receive a meaningful discount on the hourly resource usage price. The upfront fee of the reservation plan is beneficial for cloud provider, but in the long-term, the total revenue generated is lower than the one obtained by providing equivalent usage hours under an on-demand plan. The spot market pricing plan represents an auction-like mechanism, where cloud providers' customers submit the maximum price they are willing to pay. The access to cloud resources is enabled as long as offered price exceeds cloud provider's last computed market clearing price.

In order to provide content to the customers, interconnection between content provider and service provider is necessary. In this paper, three interconnection contracts with partial cloud migration are analysed: Revenue Sharing, Cost Sharing and Wholesale Price contract. Revenue Sharing contract is characterized with operational simplicity. It defines modality of revenue shares between providers involved [13]-[16]. This contract is widely implemented in cloud systems [17]-[20]. Cost Sharing contract defines cost shares among providers. This contract is analysed in [21]-[24]. Wholesale prices are established under Wholesale Price contracts and analysed in [25]-[27]. Comparison with Revenue Sharing contract is presented in [28], [29].

This paper is organized as follows. After the introductory remarks short literature review is presented in Section I. Problem statement is analysed in Section II. Modelling of traffic workload in content delivery scheme with partial cloud migration and associated interconnection contracts are described. Performance evaluation is given in Section III. 
Section IV contains discussion on obtained results. Finally, concluding remarks are given in Section V.

\section{Problem Statement}

Let us consider interconnection issues in a cloud assisted content delivery scheme depicted in Fig. 1. Participants in this scheme are: customers, service provider, content provider, cloud provider and cloud $\mathrm{CDN}$.

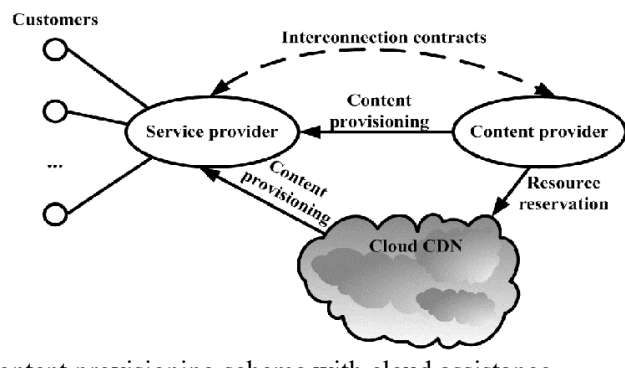

Fig. 1. Content provisioning scheme with cloud assistance.

Service provider enables connection to the customers at a flat rate retail price on a monthly basis. We assume that number of service providers' customers is fixed during observed time interval and denoted by $X$. Content provider stores all original video contents in self-owned servers, servicing major part of the requests to access certain content. Certain part of the content provider's video contents is migrated to the cloud storage. Cloud provider delivers video contents to the cloud CDN, in order to ensure satisfactory QoE. Cloud CDN delivers video contents to the service provider using a global network. Thus, the contents are delivered with the best possible performance. We assumed that cloud storage instances and cloud CDN are owned by the cloud provider. In this paper, we assign video popularity factor from the range $(0,1)$, depicting probability of access for each of video contents using Monte Carlo simulation. In accordance with the introduced content popularity factor, we estimate total bandwidth demand for access to video contents. Without general assumption violation, we perform our analysis into $N$ consecutive discrete time slots. Partial migration of content delivery to cloud is being observed. Considering limited bandwidth capacity of content provider's server, we estimate traffic delivered through cloud CDN. According to different cloud pricing schemes, revenues and assessed costs, we evaluate three interconnection pricing contracts between content and service provider.

\section{A. Modelling of Traffic Workload Characteristics}

In this paper, modelling of traffic workload is characterized at the level of communication session. A session corresponds to a request for video content provisioning. Such a modelling requires specifying a session's arrival rate and its duration. Internet traffic exhibits similar daily patterns and similar peak values every day. The number of simultaneous requests attains the highest value in the evening hours, whereas the lowest point appears in the early morning hours [10]. Being a discrete distribution, arrival rate of requests for video content provisioning is determined by Poisson distribution [2]. In each time slot requests' arrival rate is determined by Poisson distribution with parameter $\lambda_{i}, i=1, \ldots, N$.

Video content duration ranges from a few seconds to several hours. Short video contents mostly consist of advertising items. The distribution of video content duration can be approximated by an exponential distribution [30]. The session duration time is closely related to content's duration. Thus, session duration process can be approximated by an exponential distribution for the average length of the content's range.

Set of all video contents stored in content providers' selfowned servers is denoted as

$$
V_{\text {ser }}=\left\{v_{1}, v_{2}, \cdots, v_{M}\right\}
$$

For video content $v_{j}$, we introduce content popularity factor $\phi_{j}$, which indicates the probability of access to the video content $v_{j}$. It applies

$$
\phi_{j} \in(0,1), \sum_{j=1}^{M} \phi_{j}=1
$$

We further assume five quality standards for video streaming minimal required quality, recommended quality, SD quality, HD quality and Ultra HD quality, with corresponding minimal required bandwidth denoted by $w_{k}, k \in[1,5]$, respectively. Initiating request for content provisioning, customers randomly choose quality standard, according to a discrete probability distribution. The probability of choosing certain video streaming quality is denoted by $p_{k}$ and it applies $\sum_{k=1}^{5} p_{k}=1$.

Requests for video streaming in each time slot are generated according to average arrival rate. Specification of request for video streaming according to certain video content is obtained applying Monte Carlo simulation in accordance to content popularity factor. Each request corresponds to assigned bandwidth demand (depending on chosen video content quality standard) applying Monte Carlo simulation. The number of simultaneous video streams per time slot and corresponding bandwidth demand per video stream determine total bandwidth demand per time slot, denoted as $D_{i}$.

Content provider provides self-owned servers for content delivery. In order to avoid over-provisioning, servers' capacity is constrained by parameter $\alpha$. This parameter defines the portion of the peak value of total bandwidth demand per time slot. Hence, content provider's capacity can be expressed as

$$
C P_{\text {server }}=\alpha \times \max _{i}\left(D_{i}\right), \alpha \in(0,1) \text {. }
$$

Partial migration to the cloud refers to storing a portion of the most popular video contents into the cloud storage with the aim of enabling access to those contents whenever capacity of the self-owned servers is occupied. Parameter defining the replication portion of the most popular contents 
to the cloud is denoted as $\beta, \beta \in(0,1)$. In order to assure the access to the most popular video contents content provider reserves cloud storage capacity according to appropriate reservation pricing scheme. We assume that cloud storage is limited by chosen virtual machine instance, and the available cloud bandwidth is enough to satisfy all requirements during high traffic load periods. When demand exceeds capacity of the content provider's server, request for content provisioning will be accomplished if that content is being stored into the cloud. Otherwise, the request for content provisioning is rejected. Hence, portion of the contents' replication on cloud has direct impact on request rejection, especially in the periods of high traffic load.

Total number of generated requests for content provisioning per time slot is denoted by $n_{i}$. Thus, total number of generated requests can be expressed as follows

$$
n_{t}=\sum_{i=1}^{N} n_{i}=\sum_{i=1}^{N}\left(n_{i}^{c p}+n_{i}^{c l}+n_{i}^{r e j}\right)
$$

Number of request served by the content provider's server per time slot in (4) is denoted by $n_{i}^{c p}$; number of requests accomplished by the cloud CDN is denoted by $n_{i}^{c l}$ and number of rejected requests is denoted by $n_{i}^{r e j}$.

\section{B. Interconnection Contracts}

In order to analyse providers' profits, three interconnection contracts are observed: Revenue Sharing, Cost Sharing and Wholesale Price contract. In all contracts, cloud reservation pricing scheme is considered. Thus, content provider's costs can be expressed as:

$$
\begin{gathered}
C^{c p}=\sum_{i=1}^{N} C_{i}^{c p}=\sum_{i=1}^{N}\left(C_{i}^{c l}+C_{i}^{\text {ser }}\right) \\
C_{i}^{c l}=C_{i}^{\text {storage }}+C_{i}^{\text {data transfer }}
\end{gathered}
$$

where $C_{i}^{c l}$ includes content provider's costs per time slot for acquiring cloud storage for replication of the most popular video contents and costs for data transfer through cloud CDN to service provider, $C_{i}^{\text {storage }}$ consists of a fixed cost for cloud resource reservation, plus cost per time slot independent of resource usage. Cost for content provisioning from cloud CDN to service provider network is based on the transferred data volume. Total transferred data volume can be expressed as

$$
Z=\sum_{i=1}^{N} z_{i}
$$

where $z_{i}$ denotes transferred data volume per time slot. In order to obtain this cost, the following staircase function is defined

$$
C^{\text {data transfer }}=\sum_{i=1}^{N} C_{i}^{\text {data transfer }}= \begin{cases}A_{1} Z, & 0<Z \leq \gamma_{1}, \\ A_{2} Z, & \gamma_{1}<Z \leq \gamma_{2}, \\ \cdots & \\ A_{q} Z, & \gamma_{q-1}<Z \leq \gamma_{q},\end{cases}
$$

where $A_{1}, A_{2}, \cdots, A_{q}$ represent fixed prices for $q$ defined thresholds of transferred data volume when $Z$ takes values from the range $\left(\gamma_{0}, \gamma_{q}\right)$. We assume it applies $A_{1}>A_{2}>\cdots>A_{q}$.

$C_{\text {ser }}$ includes costs for self-owned servers' capacity provisioning. It can be expressed as follows

$$
C^{s e r}=C_{i}^{s e r}=\sum_{i=1}^{N} c_{m}^{c p} \times n_{i}^{c p}
$$

Marginal cost per request provisioned by content provider's self-owned servers in (9) is denoted by $c_{m}^{c p}$. Content provider's revenue is obtained as service provider's payment for interconnection establishment. Hence, service provider's cost depending of different interconnection contract- Revenue Sharing, Cost Sharing and Wholesale Price, can be represented as (10)-(12), respectively:

$$
\begin{aligned}
C_{s p}^{r s} & =\sum_{i=1}^{N} c_{m}^{s p} \times\left(n_{i}^{c p}+n_{i}^{c l}\right)+R_{c p}^{r s}, \\
C_{s p}^{c s} & =\sum_{i=1}^{N} c_{m}^{s p} \times\left(n_{i}^{c p}+n_{i}^{c l}\right)+R_{c p}^{c s}, \\
C_{s p}^{w p} & =\sum_{i=1}^{N} c_{m}^{s p} \times\left(n_{i}^{c p}+n_{i}^{c l}\right)+R_{c p}^{w p},
\end{aligned}
$$

where $c_{m}^{s p}$ denotes marginal cost per request provisioned to the customers in the service provider's network.

We assume there are $L$ possible retail flat pricing plans offered to the customers by service provider. Each pricing plan has assigned minimal guarantied bandwidth. Higher guarantied bandwidth means higher retail price $p^{r}$. Thus, service provider's revenue can be expressed as follows

$$
R_{s p}=\sum_{l=1}^{L} p_{l}^{r} \times X
$$

Content provider's revenue, depending on the interconnection contract with service provider, can be expressed as follows:

$$
\begin{gathered}
R_{c p}^{r s}=\theta \times R_{s p}, \\
R_{c p}^{c s}=(1+\rho+\tau) \times C^{c p}, \\
R_{c p}^{w p}=(1+\rho) \times C^{c p} .
\end{gathered}
$$

Parameter representing revenue share between content 
provider and service provider in Revenue Sharing contract in (14) is denoted by $\theta$; profit margin in Cost Sharing and Wholesale Price contracts in (15) and (16) is denoted by $\rho$ and parameter representing cost share in Cost Sharing contract in (16) is denoted by $\tau$. Each of these three parameters can take values in the $(0,1)$ interval.

Providers' profits can be obtained by deducting corresponding costs from revenues. Hence, content provider's profit according to corresponding interconnection contract can be written as:

$$
\begin{aligned}
\pi_{c p}^{r s} & =R_{c p}^{r s}-C^{c p}, \\
\pi_{c p}^{c s} & =R_{c p}^{c s}-C^{c p}, \\
\pi_{c p}^{w p} & =R_{c p}^{w p}-C^{c p} .
\end{aligned}
$$

Likewise, service provider's profits can be written as:

$$
\begin{gathered}
\pi_{s p}^{r s}=R_{s p}^{r s}-C_{s p}^{r s}, \\
\pi_{s p}^{c s}=R_{s p}^{c s}-C_{s p}^{c s}, \\
\pi_{s p}^{w p}=R_{s p}^{w p}-C_{s p}^{w p} .
\end{gathered}
$$

\section{PERFORMANCE EVAluAtion}

In order to evaluate model, simulations in open source programming language Python 2.7 are performed in 50 iterations. Time period of 31 days is simulated. The first day in each simulation represents warm-up period and it is not included in the simulation results. Each day is divided into 24 time slots of 1 hour duration. Total number of customers is 100000 . In order to obtain as realistic as possible traffic workload model, we used 7 different Poisson distribution parameters for arriving requests per time slot for content provisioning. The values of these parameters are: $\lambda_{1}=\lambda_{15}=\lambda_{16}=\lambda_{17}=\lambda_{18}=2.33 ; \quad \lambda_{2}=\lambda_{13}=\lambda_{14}=2$; $\lambda_{3}=\lambda_{11}=\lambda_{12}=1.67 ; \quad \lambda_{4}=\lambda_{8}=\lambda_{9}=\lambda_{10}=1.33$; $\lambda_{5}=\lambda_{6}=\lambda_{7}=1 ; \quad \lambda_{19}=\lambda_{20}=\lambda_{24}=2.67 ; \quad \lambda_{21}=\lambda_{22}=\lambda_{23}=3$. The average value for Poisson parameter is 2 requests per second, which coincide with average value in [30]. Session duration process is approximated by an exponential distribution with the average value of 30 minutes [30]. Content provider's server stores all $M=500$ original video contents. Content popularity factor is randomly assigned to each of video contents and sorted from the largest to the smallest value. Quality standards for video streaming and corresponding minimal required bandwidths are assumed according to [31]. These values are the following: minimal $0.5 \mathrm{Mb} / \mathrm{s}$, recommended $-1.5 \mathrm{Mb} / \mathrm{s}$, SD quality - $3.0 \mathrm{Mb} / \mathrm{s}$, HD quality - $5.0 \mathrm{Mb} / \mathrm{s}$ and Ultra HD quality $-25.0 \mathrm{Mb} / \mathrm{s}$. Customers randomly choose quality standard depending on constraints of access network technology. The assumed values for probability of choosing minimal, recommended, SD, HD and Ultra HD quality are: $0.15,0.20,0.30,0.20$ and 0.15 , respectively [32]. Average bandwidth demand per time slot is obtained applying Monte Carlo simulation.

Provisioning servers' capacity that satisfies total bandwidth demand is not economically efficient and the system would be in idle state most of the time. The proper bandwidth of content provider's server is from $40 \%$ to $60 \%$ of the peak demand [10]. Hence, parameter $\alpha$ in this analysis takes values: $\alpha=0.4, \alpha=0.5$ and $\alpha=0.6$. The less capacity provisioned, less resource is underutilized. Also, costs for maintaining resources are smaller. However, this may lead to greater request for content provisioning rejection, so it must be taken in consideration. A solution for this challenge might be migration to cloud resources. In order to minimize risk of content unavailability, we assume that video content migration to the cloud is occurring in the period of low traffic load, so the content uploading to the cloud would not additionally load the system. Values for the partial cloud replication parameter used in simulation are $\beta=0.2, \beta=0.4$ and $\beta=0.6$. This means that $20 \%$, $40 \%$ and $60 \%$ of the most popular video contents is replicated to the cloud. Figure 2 shows average request rejection rate depending on content provider's server capacity and the amount of content replication to the cloud. In order to assure that cloud resources are available during all observed period, we assume that content provider reserves cloud instances. This implies that content provider pays upfront fee for resource reservation according to reservation pricing scheme for corresponding instance type. Cloud providers offer various instance types and various long-term contracts. In order to obtain content provider's costs at monthly level, reservation pricing scheme and prices for data transfer through cloud CDN from [12] are observed. We assume that reserved instance type is large enough to satisfy storage requirements. Content provider's costs have the lowest value when parameter $\beta$ equals 0.2 , i.e. when migration to the cloud is the lowest, and $\alpha$ equals 0.2 , i.e. content provider serves only $40 \%$ of the peak customers' demand. However, requests' rejection rate is higher than $5 \%$ for $\beta=0.2$ and for all $\alpha$. Generally, content provider's costs increase with self-owned resources capacity enhancement, which is primary motivation for cloud migration. The appropriate combination of content provider's self-owned resources and cloud migration would be chosen in accordance with the realized profits depending on interconnection contracts with service provider.

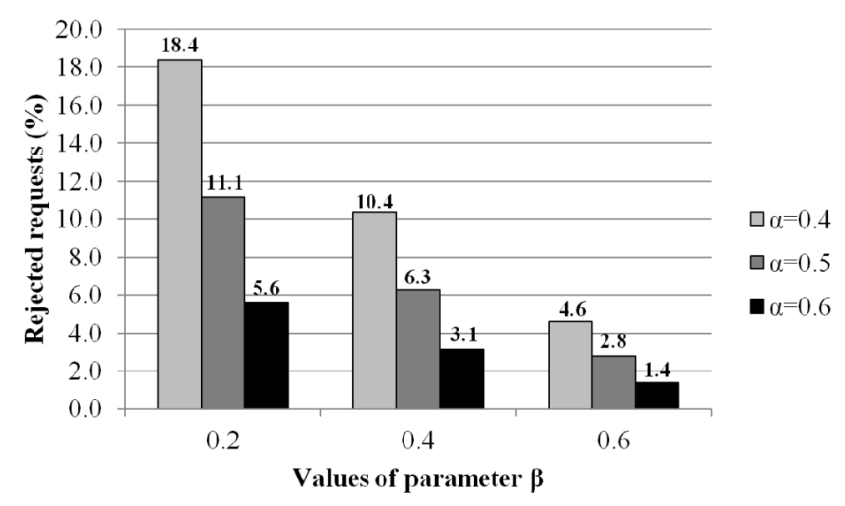

Fig. 2. Average rejection rate of requests for content provisioning.

Content provider's profits according to the Revenue Sharing, Cost Sharing and Wholesale interconnection contracts are shown in Table I, Table II and Table III, respectively. Likewise, service provider's profits obtained 
applying these interconnection contracts are shown in Table IV, Table V and Table VI, respectively.

TABLE I. CONTENT PROVIDER'S PROFITS - REVENUE SHARING CONTRACT (IN MILLION MONETARY UNITS).

\begin{tabular}{|c|c|c|c|c|c|c|}
\hline \multirow{2}{*}{$\boldsymbol{\alpha}$} & $\boldsymbol{\beta}$ & \multicolumn{6}{|c|}{ Revenue share, $\boldsymbol{\theta}(\mathbf{\% )}$} \\
\cline { 3 - 7 } & & $\mathbf{0 . 3}$ & $\mathbf{0 . 4}$ & $\mathbf{0 . 5}$ & $\mathbf{0 . 6}$ & $\mathbf{0 . 7}$ \\
\hline \multirow{3}{*}{0.4} & 0.2 & 14.028 & 31.428 & 48.828 & 66.228 & 83.628 \\
\cline { 2 - 7 } & 0.4 & 10.532 & 27.932 & 45.332 & 62.732 & 80.132 \\
\cline { 2 - 7 } & 0.6 & 8.022 & 25.422 & 42.822 & 60.222 & 77.622 \\
\hline \multirow{3}{*}{0.5} & 0.2 & 10.470 & 27.870 & 45.270 & 62.670 & 80.070 \\
\cline { 2 - 7 } & 0.4 & 8.320 & 25.720 & 43.120 & 60.520 & 77.920 \\
\cline { 2 - 7 } & 0.6 & 6.772 & 24.172 & 41.572 & 58.972 & 76.372 \\
\hline \multirow{3}{*}{0.6} & 0.2 & 7.766 & 25.166 & 42.566 & 59.966 & 77.366 \\
\cline { 2 - 7 } & 0.4 & 6.662 & 24.062 & 41.462 & 58.862 & 76.262 \\
\cline { 2 - 7 } & 0.6 & 5.867 & 23.267 & 40.667 & 58.067 & 75.467 \\
\hline
\end{tabular}

TABLE II. CONTENT PROVIDER'S PROFITS - COST SHARING CONTRACT (IN MILLION MONETARY UNITS).

\begin{tabular}{|c|c|c|c|c|c|c|}
\hline \multirow{2}{*}{$\alpha, \beta$} & \multirow{2}{*}{$\rho$} & \multicolumn{5}{|c|}{ Cost share. $\tau(\%)$} \\
\hline & & 0.1 & 0.2 & 0.3 & 0.4 & 0.5 \\
\hline \multirow{3}{*}{$\begin{array}{l}\alpha=0.4 \\
\beta=0.2\end{array}$} & 0.3 & 15.269 & 19.086 & 22.903 & 26.720 & 30.537 \\
\hline & 0.4 & 19.086 & 22.903 & 26.720 & 30.537 & 34.355 \\
\hline & 0.5 & 22.903 & 26.720 & 30.537 & 34.355 & 38.172 \\
\hline \multirow{3}{*}{$\begin{array}{l}\alpha=0.4 \\
\beta=0.4\end{array}$} & 0.3 & 16.667 & 20.834 & 25.001 & 29.168 & 33.334 \\
\hline & 0.4 & 20.834 & 25.001 & 29.168 & 33.334 & 37.501 \\
\hline & 0.5 & 25.001 & 29.168 & 33.334 & 37.501 & 41.668 \\
\hline \multirow{3}{*}{$\begin{array}{l}\alpha=0.4 \\
\beta=0.6\end{array}$} & 0.3 & 17.671 & 22.089 & 26.507 & 30.925 & 35.342 \\
\hline & 0.4 & 22.089 & 26.507 & 30.925 & 35.342 & 39.760 \\
\hline & 0.5 & 26.507 & 30.925 & 35.342 & 39.760 & 44.178 \\
\hline \multirow{3}{*}{$\begin{array}{l}\alpha=0.5 \\
\beta=0.2\end{array}$} & 0.3 & 16.692 & 20.865 & 25.038 & 29.211 & 33.384 \\
\hline & 0.4 & 20.865 & 25.038 & 29.211 & 33.384 & 37.557 \\
\hline & 0.5 & 25.038 & 29.211 & 33.384 & 37.557 & 41.730 \\
\hline \multirow{3}{*}{$\begin{array}{l}\alpha=0.5 \\
\beta=0.4\end{array}$} & 0.3 & 17.552 & 21.940 & 26.328 & 30.716 & 35.104 \\
\hline & 0.4 & 21.940 & 26.328 & 30.716 & 35.104 & 39.492 \\
\hline & 0.5 & 26.328 & 30.716 & 35.104 & 39.492 & 43.880 \\
\hline \multirow{3}{*}{$\begin{array}{l}\alpha=0.5 \\
\beta=0.6\end{array}$} & 0.3 & 18.171 & 22.714 & 27.257 & 31.799 & 36.342 \\
\hline & 0.4 & 22.714 & 27.257 & 31.799 & 36.342 & 40.885 \\
\hline & 0.5 & 27.257 & 31.799 & 36.342 & 40.885 & 45.428 \\
\hline \multirow{3}{*}{$\begin{array}{l}\alpha=0.6 \\
\beta=0.2\end{array}$} & 0.3 & 17.774 & 22.217 & 26.661 & 31.104 & 35.547 \\
\hline & 0.4 & 22.217 & 26.661 & 31.104 & 35.547 & 39.991 \\
\hline & 0.5 & 26.661 & 31.104 & 35.547 & 39.991 & 44.434 \\
\hline \multirow{3}{*}{$\begin{array}{l}\alpha=0.6 \\
\beta=0.4\end{array}$} & 0.3 & 18.215 & 22.769 & 27.323 & 31.876 & 36.430 \\
\hline & 0.4 & 22.769 & 27.323 & 31.876 & 36.430 & 40.984 \\
\hline & 0.5 & 27.323 & 31.876 & 36.430 & 40.984 & 45.538 \\
\hline \multirow{3}{*}{$\begin{array}{l}\alpha=0.6 \\
\beta=0.6\end{array}$} & 0.3 & 18.533 & 23.167 & 27.800 & 32.433 & 37.067 \\
\hline & 0.4 & 23.167 & 27.800 & 32.433 & 37.067 & 41.700 \\
\hline & 0.5 & 27.800 & 32.433 & 37.067 & 41.700 & 46.333 \\
\hline
\end{tabular}

TABLE III. CONTENT PROVIDER'S PROFITS - WHOLESALE PRICE CONTRACT (IN MILLION MONETARY UNITS).

\begin{tabular}{|c|c|c|c|c|}
\hline \multirow{2}{*}{$\boldsymbol{\alpha}$} & \multirow{2}{*}{$\boldsymbol{\beta}$} & \multicolumn{3}{|c|}{ Profit margin. $\boldsymbol{\rho}$} \\
\cline { 2 - 5 } & & $\mathbf{0 . 3}$ & $\mathbf{0 . 4}$ & $\mathbf{0 . 5}$ \\
\hline \multirow{4}{*}{0.4} & 0.2 & 11.452 & 15.269 & 19.086 \\
\cline { 2 - 5 } & 0.4 & 12.500 & 16.667 & 20.834 \\
\cline { 2 - 5 } & 0.6 & 13.253 & 17.671 & 22.089 \\
\hline \multirow{4}{*}{0.5} & 0.2 & 12.519 & 16.692 & 20.865 \\
\cline { 2 - 5 } & 0.4 & 13.164 & 17.552 & 21.940 \\
\hline & 0.6 & 13.628 & 18.171 & 22.714 \\
\hline \multirow{4}{*}{0.6} & 0.2 & 13.330 & 17.774 & 22.217 \\
\cline { 2 - 5 } & 0.4 & 13.661 & 18.215 & 22.769 \\
\hline & 0.6 & 13.900 & 18.533 & 23.167 \\
\hline
\end{tabular}

TABLE IV. SERVICE PROVIDER'S PROFITS - REVENUE SHARING CONTRACT (IN MILLION MONETARY UNITS).

\begin{tabular}{|c|c|c|c|c|c|c|}
\hline \multirow{2}{*}{$\boldsymbol{\alpha}$} & \multirow{\beta}{*}{$\boldsymbol{\beta}$} & \multicolumn{5}{|c|}{ Revenue share. $\boldsymbol{\theta}(\mathbf{\% )}$} \\
\hline \multirow{4}{*}{0.4} & 0.2 & 83.720 & 66.320 & 48.920 & 31.520 & 14.120 \\
\cline { 2 - 7 } & 0.4 & 79.974 & 62.574 & 45.174 & 27.774 & 10.374 \\
\cline { 2 - 7 } & 0.6 & 77.285 & 59.885 & 42.485 & 25.085 & 7.685 \\
\hline \multirow{4}{*}{0.5} & 0.2 & 80.345 & 62.945 & 45.545 & 28.145 & 10.745 \\
\cline { 2 - 7 } & 0.4 & 78.079 & 60.679 & 43.279 & 25.879 & 8.479 \\
\cline { 2 - 7 } & 0.6 & 76.446 & 59.046 & 41.646 & 24.246 & 6.846 \\
\hline \multirow{3}{*}{0.6} & 0.2 & 77.745 & 60.345 & 42.945 & 25.545 & 8.145 \\
\cline { 2 - 7 } & 0.4 & 76.610 & 59.210 & 41.810 & 24.410 & 7.010 \\
\cline { 2 - 7 } & 0.6 & 75.795 & 58.395 & 40.995 & 23.595 & 6.195 \\
\hline
\end{tabular}

TABLE V. SERVICE PROVIDER'S PROFITS - COST SHARING CONTRACT (IN MILLION MONETARY UNITS).

\begin{tabular}{|c|c|c|c|c|c|c|}
\hline \multirow{2}{*}{$\alpha, \beta$} & \multirow{2}{*}{$\rho$} & \multicolumn{5}{|c|}{ Cost share. $\tau(\%)$} \\
\hline & & 0.1 & 0.2 & 0.3 & 0.4 & 0.5 \\
\hline \multirow{3}{*}{$\begin{array}{l}\alpha=0.4 \\
\beta=0.2\end{array}$} & 0.3 & 82.480 & 78.663 & 74.846 & 71.028 & 67.211 \\
\hline & 0.4 & 78.663 & 74.846 & 71.028 & 67.211 & 63.394 \\
\hline & 0.5 & 74.846 & 71.028 & 67.211 & 63.394 & 59.577 \\
\hline \multirow{3}{*}{$\begin{array}{l}\alpha=0.4 \\
\beta=0.4\end{array}$} & 0.3 & 73.839 & 69.672 & 65.506 & 61.339 & 57.172 \\
\hline & 0.4 & 69.672 & 65.506 & 61.339 & 57.172 & 53.005 \\
\hline & 0.5 & 65.506 & 61.339 & 57.172 & 53.005 & 48.838 \\
\hline \multirow{3}{*}{$\begin{array}{l}\alpha=0.4 \\
\beta=0.6\end{array}$} & 0.3 & 67.636 & 63.218 & 58.800 & 54.382 & 49.964 \\
\hline & 0.4 & 63.218 & 58.800 & 54.382 & 49.964 & 45.547 \\
\hline & 0.5 & 58.800 & 54.382 & 49.964 & 45.547 & 41.129 \\
\hline \multirow{3}{*}{$\begin{array}{l}\alpha=0.5 \\
\beta=0.2\end{array}$} & 0.3 & 74.123 & 69.950 & 65.777 & 61.603 & 57.430 \\
\hline & 0.4 & 69.950 & 65.777 & 61.603 & 57.430 & 53.257 \\
\hline & 0.5 & 65.777 & 61.603 & 57.430 & 53.257 & 49.084 \\
\hline \multirow{3}{*}{$\begin{array}{l}\alpha=0.5 \\
\beta=0.4\end{array}$} & 0.3 & 68.846 & 64.458 & 60.070 & 55.682 & 51.294 \\
\hline & 0.4 & 64.458 & 60.070 & 55.682 & 51.294 & 46.906 \\
\hline & 0.5 & 60.070 & 55.682 & 51.294 & 46.906 & 42.518 \\
\hline \multirow{3}{*}{$\begin{array}{l}\alpha=0.5 \\
\beta=0.6\end{array}$} & 0.3 & 65.047 & 60.504 & 55.961 & 51.419 & 46.876 \\
\hline & 0.4 & 60.504 & 55.961 & 51.419 & 46.876 & 42.333 \\
\hline & 0.5 & 55.961 & 51.419 & 46.876 & 42.333 & 37.790 \\
\hline \multirow{3}{*}{$\begin{array}{l}\alpha=0.6 \\
\beta=0.2\end{array}$} & 0.3 & 67.737 & 63.293 & 58.850 & 54.406 & 49.963 \\
\hline & 0.4 & 63.293 & 58.850 & 54.406 & 49.963 & 45.520 \\
\hline & 0.5 & 58.850 & 54.406 & 49.963 & 45.520 & 41.076 \\
\hline \multirow{3}{*}{$\begin{array}{l}\alpha=0.6 \\
\beta=0.4\end{array}$} & 0.3 & 65.058 & 60.504 & 55.950 & 51.397 & 46.843 \\
\hline & 0.4 & 60.504 & 55.950 & 51.397 & 46.843 & 42.289 \\
\hline & 0.5 & 55.950 & 51.397 & 46.843 & 42.289 & 37.735 \\
\hline \multirow{3}{*}{$\begin{array}{l}\alpha=0.6 \\
\beta=0.6\end{array}$} & 0.3 & 63.128 & 58.495 & 53.862 & 49.228 & 44.595 \\
\hline & 0.4 & 58.495 & 53.862 & 49.228 & 44.595 & 39.962 \\
\hline & 0.5 & 53.862 & 49.228 & 44.595 & 39.962 & 35.328 \\
\hline
\end{tabular}

TABLE VI. SERVICE PROVIDER'S PROFITS - WHOLESALE PRICE CONTRACT (IN MILLION MONETARY UNITS).

\begin{tabular}{|c|c|c|c|c|}
\hline \multirow{3}{*}{$\boldsymbol{\alpha}$} & $\boldsymbol{\beta}$ & \multicolumn{3}{|c|}{ Profit margin. $\boldsymbol{\rho}$} \\
\cline { 2 - 5 } & & $\mathbf{0 . 3}$ & $\mathbf{0 . 4}$ & $\mathbf{0 . 5}$ \\
\hline \multirow{3}{*}{0.4} & 0.2 & 86.297 & 82.480 & 78.663 \\
\cline { 2 - 5 } & 0.4 & 78.006 & 73.839 & 69.672 \\
\cline { 2 - 5 } & 0.6 & 72.053 & 67.636 & 63.218 \\
\hline \multirow{3}{*}{0.5} & 0.2 & 78.296 & 74.123 & 69.950 \\
\cline { 2 - 5 } & 0.4 & 73.234 & 68.846 & 64.458 \\
\cline { 2 - 5 } & 0.6 & 69.590 & 65.047 & 60.504 \\
\hline \multirow{3}{*}{0.6} & 0.2 & 72.180 & 67.737 & 63.293 \\
\cline { 2 - 5 } & 0.4 & 69.612 & 65.058 & 60.504 \\
\cline { 2 - 5 } & 0.6 & 67.762 & 63.128 & 58.495 \\
\hline
\end{tabular}

IV. DISCUSSION

Simulation results for providers' profits and possible 
cloud migration obtained applying all observed interconnection contracts show regularity in profit values for all resource combinations. Analysis of requests' rejection rate and occupied resources indicates that cloud migration is desirable in all observed situations. Costs' analysis confirms this conclusion. In accordance with, for all observed values of parameters $\alpha$ and $\beta$, Revenue Sharing contract assures the greatest profits for content provider, with revenue share of $70 \%, 60 \%$ and $50 \%$. The next acceptable solution for content provider is Cost Sharing contract with cost share of $50 \%$ and profit margin $40 \%$ and $50 \%$, or cost share of $40 \%$ and profit margin of $50 \%$. Analysing remaining contractual conditions, Revenue sharing with revenue share of $40 \%$ achieves higher profits of all other solutions. It is worth noting that Wholesale Price contract achieves very low profits in comparison with all other contracts. Service provider's position is completely opposite from content provider. The following consideration is valid for all combinations of content provider's self-owned resources and cloud migration. It is evident that all observed contracts assure that service provider never operates with losses. Hence, any contractual form enables costs recovery. Wholesale Price contract achieves high profits in comparison with other contracts. As expected, Revenue Sharing with revenue share of $50 \%, 60 \%$ and $70 \%$ provides the lowest profits. If service provider sets its profit margin at, for instance, $30 \%$, these revenue shares will not meet the requirements. Cost Sharing contracts representing second best solution for content provider also will not be acceptable from service provider's perspective. However, Revenue Sharing contract with revenue share of $40 \%$ satisfies service provider profit margin constraint. Observation of service and content provider's profits infer that Revenue Sharing contract with revenue share of $40 \%$ can be considered as a medium-effective solution for both providers.

\section{CONCLUSIONS}

This paper presents observation of traffic workload under uncertainty of demand in content provisioning system with partial cloud migration. We analyse bandwidth demand, resource utilization, requests' rejection rate, cost for partial cloud migration and providers' profits obtained under different interconnection contracts. Bandwidth demand is estimated using Monte Carlo simulation with content popularity factor as a relevant parameter. It is notable that provisioning of resources that satisfy peak bandwidth demand leads to low utilization. However, underprovisioning increases average requests' rejection rate. This paper shows that partial cloud migration can be costeffective solution for that challenge. It also decreases average requests' rejection rate. Analysis of this system for content provisioning with cloud assistance is extended in order to obtain a proper interconnection contract between content and service provider. It approves that Revenue Sharing can assure satisfying profits for both providers.

\section{REFERENCES}

[1] BEREC Report. BoR (12) 130, 2012.
[2] E. Harstead, R. Sharpe, "Forecasting of access network bandwidth demands for aggregated subscribers using Monte Carlo methods", IEEE Communications Magazine, vol. 53, no. 3, pp. 199-207, 2015. [Online]. Available: https://doi.org/10.1109/MCOM.2015.7060505

[3] Cisco Visual Networking Index. 2015. [Online]. Available: http://www.cisco.com/c/en/us/solutions/collateral/service-provider/ipngn-ip-next-generation-network/white_paper_c11-481360.pdf [Online]. Available: https://doi.org/10.4159/harvard.9780674434929

[4] Z. Avramova. S. Witevrongel. H. Bruneel, D. De Vleeschauwer, "Analysis and modeling of video popularity evolution in various online video content systems: power-law versus exponential decay", in Proc. 2009 First Int. Conf. Evolving Internet, Cannes, 2009, pp. 95-100. [Online]. Available: https://doi.org/10.1109/INTERNET. 2009.22

[5] J. K. Zipf, Selective Studies and the Principle of Relative Frequency in Language. Harvard University Press. Cambridge. Massachusetts. 1932.

[6] W. Tang. Y. Fu. L. Cherkasova, A. Vahdat, "Modeling and generating realistic streaming media server workloads", Computer Networks, vol. 51. no. 1. pp. 336-356, 2007. [Online]. Available: https://doi.org/10.1016/j.comnet.2006.05.003

[7] L. Guo, E. Tan, S. Chen, Z. Xiao, X. Zhang, "The stretched exponential distribution of internet media access patterns", in Proc 27th ACM Symposium on Principles of Distributed Computing, New York, 2008, pp. 283-294. [Online]. Available: https://doi.org/ $10.1145 / 1400751.1400789$

[8] M. Cha, H. Kwak, P. Rodriguez, Y-Y. Ahn, S. Moon, "I Tube, You Tube, Everybody Tubes: analyzing the world's largest user generated content video system", in Proc. 7th ACM SIGCOMM Conf. Interne Measurements, New York, 2007, pp. 1-14. [Online]. Available: https://doi.org/10.1145/1298306.1298309

[9] P. Jamashidi, A. Ahmad, C. Pahl, "Cloud migration research: a systematic review", IEEE Trans. Cloud Computing, vol. 1, no. 2, pp. 142-157, 2013. [Online]. Available: https://doi.org/10.1109/ TCC.2013.10

[10] H. Li, J. Liu, B. Li, K. Xu, "Cost-effective partial migration of VoD services to content clouds", in Proc. 2011 IEEE 4th Int. Conf. Cloud Computing, Washington, 2011, pp. 203-210. [Online]. Available: https://doi.org/10.1109/cloud.2011.41

[11] X. Qiu, H. Li, C. Wu, Z. Li, F. C. M. Lau, "Cost-minimizing dynamic migration of content distribution services into hybrid clouds", in Proc. 2012 IEEE INFOCOM, Orlando, 2012, pp. 2571-2575.

[12] Amazon EC2 pricing. 2015. [Online]. Available: https://aws.amazon.com/ec2/pricing/

[13] Y. Wu, H. Kim, P. H. Hande, M. Chiang, D. H. K. Tsang, "Revenue sharing among ISPs in two-sided markets", in Proc. 2011 IEEE INFOCOM, Shanghai, 2011, pp. 596-600.

[14] H. Lee, H. Jang, Y. Yi, J. Cho, "On the interaction between contentoriented traffic scheduling and revenue sharing among providers", in Proc. 2013 IEEE INFOCOM, Turin, 2013, pp. 3201-3206.

[15] P. Coucheney, P. Maille, B. Tuffin, "Network neutrality debate and ISP inter-relations: traffic exchange, revenue sharing, and disconnection threat", Economics Research and Electronic Networking, vol. 15, no. 3, pp. 155-182, 2014. [Online]. Available: https://doi.org/10.1007/s11066-014-9090-3

[16] L. He, J. Walrand, "Pricing and revenue sharing strategies for internet service providers", IEEE Journal on Selected Areas in Communications, vol. 24, no. 5, pp. 942-951, 2006. [Online] Available: https://doi.org/10.1109/JSAC.2006.872875

[17] L. Wei, X. Yang, "Revenue sharing contract in a cloud computing service supply chain with a monopoly AIP and multiple competing ASPs under asymmetric information", in Proc. 2015 12th Int. Conf. on Service Systems and Service Management, Guangzhou, 2015, pp. 1-6.

[18] B. E. Zant, I. Amigo, M. Gagnaire, "Federation and revenue sharing in cloud computing environment", in Proc. 2014 IEEE Int. Conf. Cloud Engineering, Boston, 2014, pp. 11-14. [Online]. Available: https://doi.org/10.1109/IC2E.2014.78

[19] J. Cohen, L. Echabbi, "Pricing composite cloud services: the cooperative perspective", in Proc. 2014 Fifth Int. Conf. next Generation Networks and Services, Casablanca, 2014, pp. 335-342. [Online]. Available: https://doi.org/10.1109/ngns.2014.6990274

[20] M. M. Hassan, M. A. Al-Wadud, G. Fortino, "A socially optimal resource and revenue sharing mechanism in cloud federations", in Proc. 2015 IEEE 19th Int. Conf. Computer Supported Cooperative Work in Design (CSCWD), Calabria, 2015, pp. 620-625. [Online] Available: https://doi.org/10.1109/cscwd.2015.7231029 
[21] A. Gupta, A. Kumar, M. Pa'L, T. Roughgarden, "Approximation via cost sharing: simpler and better approximation algorithms for network design", Journal of the ACM, vol. 54, no. 3, pp. 1-39, 2007.

[22] A. Bogomolnaia, R. Holzman, H. Moulin, "Sharing the cost of a capacity network", Mathematics of Operational Research, vol. 35, no. 1, pp. 173-192, 2010. [Online]. Available: https://doi.org/ $10.1287 /$ moor. 1090.0435

[23] D. L'opez-Pintado, "Network formation, cost-sharing and anticoordination", Int. Game Theory Review, vol. 11, no. 1, pp. 53-76, 2009. [Online]. Available: https://doi.org/10.1142/S02191989 09002145

[24] M. Hoefer, "Strategic cooperation in cost sharing games", Int. J. Game Theory, vol. 42, no. 1, pp. 29-53, 2013. [Online]. Available: https://doi.org/10.1007/s00182-011-0312-8

[25] J. D. Song, "Various wholesale price equilibria for mobile virtual network operators", Telecommunication Policy, vol. 34 , no. 10 pp. 633-648, 2010. [Online]. Available: https://doi.org/10.1016/ j.telpol.2010.07.002

[26] Y. Xu, A. Bisi, "Wholesale-price contracts with postponed and fixed retail prices", Operational Research Letters, vol. 40, no. 4, pp. 250257, 2012. [Online]. Available: https://doi.org/10.1016/j.orl. 2012.04.001
[27] X. Wang, X. Wang, Y. Su, "Wholesale-price contract of supply chain with information gathering", Applied Mathematical Modelling, vol. 37, no. 6, pp. 3848-3860, 2013. [Online]. Available: https://doi.org/10.1016/j.apm.2012.07.007

[28] K. Pan. K. K. Lai, S. C. H. Leung, D. Xiao, "Revenue-sharing versus wholesale price mechanisms under different channel power structures", European Journal of Operational Research, vol. 203, no. 2, pp. 532-538, 2010. [Online]. Available: https://doi.org/ 10.1016/j.ejor.2009.08.010

[29] Y. Gerchak, Y. Wang, "Revenue-sharing vs. wholesale-price contracts in assembly systems with random demand", Production and Operations Management, vol. 13, no. 1, pp. 23-33, 2004. [Online]. Available: https://doi.org/10.1111/j.1937-5956.2004.tb00142.x

[30] Y. Choi. J. A. Silvester, H. Kim, "Analyzing and modelling workload characteristics in a multiservice IP network", IEEE Internet Computing, vol. 15 , no. 2, pp. 35-42, 2011. [Online]. Available: https://doi.org/10.1109/MIC.2010.153

[31] Netflix internet connection speed recommendations. [Online]. Available: https://help.netflix.com/en/node/306

[32] An Overview of the Telecom and Postal Services Market in the Republic of Serbia in 2014, RATEL, 2015. 